\title{
La position générique du Crabro tabanicida Fischer |Hym.
}

\section{Sphecidae]}

\section{Jean Leclercq}

\section{Citer ce document / Cite this document :}

Leclercq Jean. La position générique du Crabro tabanicida Fischer |Hym. Sphecidae]. In: Bulletin de la Société entomologique de France, volume 56 (7), juillet 1951. pp. 105-106;

https://www.persee.fr/doc/bsef_0037-928x_1951_num_56_7_18518

\section{Ressources associées :}

Crabro tabanicida

Sphecidae

Fichier pdf généré le 04/07/2019 
Guignot (F.). - Les Hydrocanthares de France, 1931-1933.

Coléoptères Hydrocanthares, Faune de France, 48, 1947.

Legros (C.). - Essai sur les tourbières à Sphaignes des environs de Paris. L'Entomologiste, T. I, fasc. 3-4, 1945, pp. 49-55.

Mayet (V.). - Sur la arve de l'Eunectes sticticus L. Ann. Soc. Entom. France, Bull. 1887, pp. CCIII-CCIV.

Meinert (F.). - Larvae Dytiscidarum (Vandkalvelarvene). Det kongelige Danske Vidensk. Selsk. Skrift. Natur, math., Afd. IV, 1901.

Nowrojee (D.). -- Life historics of Indian Insects, 11, Tome aquatic Rhynchota and Coleoptera, Ind. Dep. Agr. India, Entom. Ser., II, n $19,1912$.

\title{
La position générique du Crabro tabanicida Fischer
}

\author{
[Hy.m. SpHecidan] \\ par Jean Leclerce
}

Le Crabro tabanicida (Fischer, 1929) est indiscutablement un Ectemnius, au sens de V.S.L. Pate (1944, etc.). La diagnose originale et les dessins en couleur qui l'accompagnent ne laissent aucun doute à ce sujet. Mais cet Ectemnius est le seul à posséder 13 articles aux entennes chez le $\hat{b}$. Considérant ce caractère, on serait tenté de l'apparenter aux Williamsita de la faune australienne, mais l'absence d'aire pygidiale chez le $\hat{o}$, la présence d'une forte dent interne aux mandibules, la forme des premiers articles du funicule et la largeur du premier segment abdominal à la base, sont autant d'objections à cette hypothèse. Par contre, les caractères précités et la structure générale du corps conduisent à penser que le Crabro tabanicida est à rapprocher des Ectemnius des sous-genres Hypocrabro et Apoctemnius. La pigmentation, notamment, est tout à fait semblable a celle qui est de règle chez les Apoctemnius (Leclercq, 1950). Il paraît donc préférable de créer un sous-genre nouveau pour recevoir cette espèce si particulière.

Sous-genre Protoctemnius, nov.

(Générotype: Crabro tabanicida Fischer, C.R., 1929, p. 43; 1929, p. 157 ( \&, ô ; Brésil: Sâo Paulo; prédateur de Diptères Tabanides).

Taille et aspect général des Ectemnius, notamment des Apoctemnius Leclercq (1950). Antennes de 13 articles che le $\hat{o}$, de 12 chez la $\circ$. Les articles basilaires du funicule ne sont ni échancrés, ni denticulés, ni anormalement allongés (le $2^{\mathrm{c}}$ est moins long que le $1^{\text {er }}+$ le $3^{\text {, }}$, chez les deux sexes). Mandibules bifides à l'extrémité, avec une forte dent interne $\left(\begin{array}{c}\delta \\ \delta\end{array}\right)$. Ocelles disposés en triangle très aplati. Mésonotum densément chagriné-ponctué; mésopleures ponctuées avec des rides dans la partie supéricure. Surface du segment médiaire rugueuse, avec un sulcus longitudinal médian et une aire dorsale assez bien définie. Ailes postérieures normales. Pattes sans modifications, même chez le $\delta$. Tibias II avec un éperon ( () , sans éperon distinct ( $\hat{o})$. Abdomen parfaitement sessile, à ponctuation fine sur les tergites. Pas d'aire pygidiale $\delta$; aire pygidiale $q$ allongée, creusie en gouttière et fortement amincie vers l'apex. Corps noir avec des parties 
jaunes, y compris des bandes plus ou moins interrompues sur les tergites. La o nidifie dans les vieilles branches et approvisionne ses cellules de Diptères Tabanides adultes. La seule espèce connue vit au Brésil, dans la région de Sâo Paulo.

Il y aurait lieu de préciser ultérieurement les particularités relatives aux caractères suivants, non mentionnés dans la diagnose originale du Crabro tabanicida: Présence d'une carène aux scapes, fossettes supra-orbitale, forme de la carène occipitale, de la carène qui précède les hanches II et structure de la partic inférieure des mésopleures, lobe anal des ailes pestérieures et présence éventuelle d'impressions transversales à la base des tergites.

\title{
Université de Liège (Biochimie) et Institut Royal des Sciences Naturelles de Belgique
}

\section{Bribliographe}

Fiscier (C.R.) 1929. - De un nuevo Himenoptero destruitor de motucas. (Bol. Biol., Sâo Paulo, n" 15,43.)

Fischer (C. R.) 1929. - Nolas biologicas sobre o Crabro tabanicida Fischer, 1929, e consideracoes concernentes as motucas. (Arqu. Inst. Biol., Sâo Paulo, II, 141.)

Lectunce (J.) 1950. - Les Ectemnius sud-américains du sous-genre Apoctemnius. (Rev. Fr. Entom., XVII, 200).

PATE (V.S.I..) 1944. -- Conspectus of the genera of Pemphilidine Wasps. (Amer. Midland Nat., XXXI, 329.)

\section{Nouvelles stations du Palpigrade Koenenia mirabilis Grassi}

\author{
par Paul A. Rемy
}

Cet Arachnide, rencontré en de nombreuses stations d'Europe méridionale (France, Corse, Italie, Sicile, Corfou) et d'Afrique du Nord (Maroc, Algérie, Tunisie, Egypte), ainsi qu'au Liban et à Madagascar (région de Tananarive), n'avait pas encore été mentionné de la péninsule ibérique.

Je viens d'en déterminer $4 \%$ adultes que le Dr A. DE Barros Machado a eu l'obligeance de récolter à mon intention au Portugal, à Porto: 2, longues respectivement de 0,85 et $0,90 \mathrm{~mm}$., le 25 mars $1951 ; 2$, longues de 0,71 et $0,77 \mathrm{~mm}$., le 15 avril $1951^{1 .}$.

D'autre part, j'ai récolté de nouveaux spécimens algériens de l'espèce au cours d'un voyage d'Alger au Hoggar et retour, en mars et avril 1951.

Blida: $a$ ) jardin Bizot, 3 \& adultes, longues de $0,77,0,80$ et $0,86 \mathrm{~mm}$., et 2 jeunes de 0,48 et $0,59 \mathrm{~mm}$; $b$ ) parc d'une villa, rive gauche de l'oued el Kebir à sa sortie des gorges, 1 \& adulte de $0,96 \mathrm{~mm}$.

Bou Saada: jardins près du pavillon de Dinet, 2 . adultes de 0,66 et $0,77 \mathrm{~mm}$.

(1) Ces dernières proviennent du jardin du Palacio de Cristal; la station des 2 autres ne m’est pas précisée. 